\title{
Dor social em pacientes oncológicos elegíveis aos cuidados paliativos na atenção
}

\section{domiciliar: Revisão integrativa}

\author{
Social pain in oncological patients eligible to palliative care in home care: Integrative review \\ Dolor social en pacientes oncológicos elegibles para la atención paliativa en la atención en el hogar:
}

\section{Revisión integrativa}

Recebido: 12/03/2021 | Revisado: 21/03/2021 | Aceito: 18/04/2021 | Publicado: 29/04/2021

\author{
Alexandre Ernesto Silva \\ ORCID: https://orcid.org/0000-0001-9988-144X \\ Universidade Federal de São João Del Rei, Brasil \\ E-mail: alexandresilva@ufsj.edu.br \\ Gabriel Alves Silva \\ ORCID: https://orcid.org/0000-0003-4956-343X \\ Universidade Federal de São João Del Rei, Brasil \\ E-mail: gabriel.silva989@outlook.com \\ Kariny Aparecida Trevisan da Silva \\ ORCID: https://orcid.org/0000-0003-0704-368X \\ Universidade Federal de São João Del Rei, Brasil \\ E-mail: karinyatsenf@gmail.com \\ Mariana Ferreira dos Santos \\ ORCID: https://orcid.org/0000-0001-7634-9733 \\ Universidade Federal de São João Del Rei, Brasil \\ E-mail: marianaferreiradossantos25@gmail.com \\ Thayane Vieira Carvalho \\ ORCID: https://orcid.org/0000-0001-8644-8954 \\ Clínica Auge, Brasil \\ E-mail: thayanevieira100@yahoo.com.br \\ Stefãne Amorim Ribeiro \\ ORCID: https://orcid.org/0000-0001-9702-1913 \\ Faculdade Asa de Brumadinho, Brasil \\ E-mail: stefanearibeiro@yahoo.com.br
}

\begin{abstract}
Resumo
Introdução: A prevalência de doenças crônicas não transmissíveis (DCNT) tem aumentado nas últimas décadas, e as neoplasias aparecem em segundo lugar merecendo destaque no cenário atual. A experiência de troca de papel social acompanhada de sentimentos de ansiedade, abandono e solidão denomina-se dor social, a qual na maioria das vezes acompanha o paciente oncológico. Este artigo tem como objetivo conhecer e compreender as causas da dor social em pacientes oncológicos elegíveis aos cuidados paliativos domiciliares. Metodologia: Trata-se de uma revisão integrativa de literatura com busca bibliográfica dos últimos cinco anos, na base de dados PUBMED, utilizando a seguinte questão norteadora de pesquisa: Quais as causas da dor social de pessoas com câncer elegíveis aos cuidados paliativos na atenção domiciliar? Resultados: Após análise dos critérios, foram definidos 3 artigos para a revisão por preencherem os critérios de seleção. Conclusão: As causas da dor social envolvem as atividades de vida diária, troca de papeis, isolamento e solidão. O cuidado paliativo e a atenção domiciliar têm um papel valioso para abordar, prevenir e proporcionar o alívio da dor social, porém, existe uma carência de conhecimento por parte dos profissionais de saúde sobre essa temática, o que pode ser melhor abordado nas instituições de ensino.
\end{abstract}

Palavras-chave: Cuidados paliativos; Serviços de assistência domiciliar; Solidão.

\begin{abstract}
Introduction: The prevalence of chronic non-communicable diseases (CNCDs) has increased in recent decades, and neoplasms appear in second place deserving prominence in the current scenario. The experience of changing social roles accompanied by feelings of anxiety, abandonment and loneliness is called social pain, which most often accompanies the cancer patient. This article aims to know and understand the causes of social pain in cancer patients eligible for home palliative care. Methodology: This is an integrative literature review with bibliographic search of the last five years, in the PUBMED database, using the following research guiding question: What are the causes of social pain of people with cancer eligible for palliative care in home care? Results: After analyzing the criteria, 3 articles were defined for review because they met the selection criteria. Conclusion: The causes of social pain involve activities of daily living, changing roles, isolation and loneliness. Palliative care and home care play a valuable role in
\end{abstract}


addressing, preventing and providing relief from social pain, however, there is a lack of knowledge on the part of health professionals on this topic, which can be better addressed in educational institutions.

Keywords: Palliative care; Home care services; Loneliness.

\section{Resumen}

Introduccion: La prevalencia de enfermedades crónicas no transmisibles (ECNT) se ha incrementado en las últimas décadas, y las neoplasias aparecen en un segundo lugar merecedor de protagonismo en el escenario actual. La experiencia de cambiar los roles sociales acompañada de sentimientos de ansiedad, abandono y soledad se llama dolor social, que con mayor frecuencia acompaña al paciente con cáncer. Este artículo tiene como objetivo comprender y comprender las causas del dolor social en pacientes con cáncer elegibles para cuidados paliativos domiciliarios. Metodología: Se trata de una revisión integradora de la literatura con búsqueda bibliográfica de los últimos cinco años, en la base de datos PUBMED, utilizando la siguiente pregunta orientadora de la investigación: ¿Cuáles son las causas del dolor social de las personas con cáncer elegibles para cuidados paliativos en el hogar? Resultados: Tras analizar los criterios, se definieron 3 artículos para revisión por cumplir con los criterios de selección. Conclusión: Las causas del dolor social involucran actividades de la vida diaria, cambios de roles, aislamiento y soledad. Los cuidados paliativos y los cuidados domiciliarios juegan un papel valioso en el abordaje, prevención y alivio del dolor social, sin embargo, existe un desconocimiento por parte de los profesionales de la salud sobre este tema, que puede ser mejor abordado en las instituciones educativas.

Palabras clave: Cuidados paliativos; Servicios de atención de salud a domicilio; Soledad.

\section{Introdução}

A prevalência de doenças crônicas não transmissíveis (DCNT) tem aumentado nas últimas décadas e o crescimento dos hábitos de vida que comprometem a saúde tem levado as DCNT ao topo das causas de mortalidade geral nos últimos anos. Essas doenças dominam as três primeiras causas de mortalidade geral, de modo que, as neoplasias aparecem em segundo lugar, perdendo apenas para as doenças cardiovasculares. Em 2020, segundo dados do Instituto Nacional do câncer (INCA), 309.750 homens e 316.280 mulheres, morreram devido doenças neoplásicas, totalizando 626,03 óbitos por neoplasia em 1 ano (DATASUS, 2019; INCA, 2020).

As Neoplasias são doenças causadas pelo crescimento desordenado de células modificadas. Essas células modificadas podem ter crescimento tumoral ou infiltrativo, sendo essas últimas relacionadas aos casos mais graves dessas doenças. (INCA, 2020). Uma das manifestações clínicas mais características do câncer é a dor. A dor experimentada por um paciente oncológico passa por todas as dimensões e particularidades dessa, sendo a dor física a mais comum e com maior gama de tratamentos disponíveis (Crofford, 2015).

A dor é uma experiência subjetiva, individual, física, espiritual e/ou emocional desagradável relacionada a dano ao corpo. A fisiopatologia da dor física envolve a condução de impulsos nervosos e respostas a estímulos, porém existem dores que não estão associadas à resposta biológica. A dor tem três dimensões importantes: sensorial, onde e quando dói, a emocional, quanto a experiência é desagradável e cognitiva, como essa dor é interpretada e quais suas consequências (Crofford, 2015).

Vale destacar que há um número cada vez maior de mortes decorrentes de doenças crônicas ou progressivas, aumentando de forma considerável o percentual de doentes em estágios terminais em hospitais e domicílios. Diante disso, os cuidados paliativos se tornaram cada vez mais essenciais e deveriam ser oferecidos em todos os sistemas de saúde (Roque, Silva, Santos, Sousa \& Perim, 2020).

O curso natural do câncer leva, por vezes, o paciente à completa dependência de cuidados, e, nesses casos, se torna um desafio manter a qualidade de vida dessas pessoas. As mudanças provocadas pelo avanço da condição patológica geralmente levam à ansiedade e uma troca nas funções sociais daquela pessoa. A experiência de troca de papel social acompanhada de sentimentos de abandono, ansiedade e solidão denomina-se dor social (Crofford, 2015).

A dor social é relatada em diversos casos oncológicos, porém, é melhor percebida em situações que a doença ameaça a continuidade da vida e que pacientes e familiares necessitam de cuidados paliativos. Os cuidados paliativos oferecem um 
suporte para pacientes com doenças graves por meio da manutenção da qualidade de vida e o respeito aos direitos humanos (Sarmento, Gysels, Higginson \& Gomes, 2016).

Essa abordagem tem como um de seus princípios o controle da dor e de outros sintomas desagradáveis, incluindo as dimensões física, psicossocial, espiritual e familiar. Dentre os critérios de elegibilidade aos cuidados paliativos, estão presentes as doenças graves, progressivas e incuráveis que ameacem a continuidade da vida (OMS, 2002). Dentre essas doenças destacam os diversos tipos de câncer, que se caracteriza como doença grave, potencialmente incurável e que em muitas situações prognósticas ameaçam a continuidade da vida (Vasconcelos \& Pereira, 2018).

Considerando as inúmeras formas de organizações de serviços para pacientes que necessitam de cuidados paliativos, ressaltando aqui os oncológicos, em um cenário que promove conforto e acolhimento, destacam-se os serviços de atenção domiciliar (Souza \& Kerbauy, 2017). A abordagem em atenção domiciliar para pacientes com câncer tem relação positiva com o aumento da qualidade de vida. A associação de cuidados paliativos e atenção domiciliar como uma abordagem de cuidado mostra-se uma ferramenta eficaz para que a dor não física do paciente oncológico seja controlada, mediante o conhecimento das possíveis origens dessas dores.

Mediante essa contextualização, o presente estudo tem como objetivo conhecer e compreender as causas da dor social em pacientes oncológicos elegíveis aos cuidados paliativos domiciliares.

\section{Metodologia}

Trata-se de uma revisão integrativa da literatura, de caráter qualitativo, com coleta de dados realizada a partir de fontes secundárias de uma investigação bibliográfica que se desenvolveu por meio de cinco etapas conforme recomendado por (Mendes, Silveira \& Galvão, 2008). A primeira etapa é composta pela identificação do tema e a escolha da questão de pesquisa; na segunda etapa é realizada uma busca e seleção na literatura com os critérios de inclusão/exclusão; na terceira etapa definem-se estudos para que na próxima etapa seja executada uma avaliação dos estudos pré-selecionados e por fim uma interpretação dos resultados e discussão que resultou na seguinte questão de pesquisa: Quais as causas da dor social de pessoas com câncer em cuidados paliativos na atenção domiciliar?

Na segunda etapa foram utilizados Descritores em Ciências da Saúde (DeCS). As seguintes estratégias de busca foram aplicadas para seleção das produções científicas. O operador booleano AND para buscar maior quantitativo de publicações no sistema de bases PUBMED. Foram utilizados como descritores: palliative care, home care e loneliness, com o operador booleano AND.

Os critérios de inclusão definidos para a seleção dos artigos foram: artigos publicados entre 2015 e 2020, artigos na íntegra e grátis na plataforma PUBMED, e artigos diretamente relacionados ao tema nos idiomas português, inglês e espanhol. Os critérios de exclusão foram artigos pagos, publicados em outros idiomas, artigos de revisão, resenhas e relatos de experiência.

A terceira etapa foi realizada com a elaboração de uma tabela no programa Microsoft Word 2010® para organizar os artigos escolhidos contendo ano, estudo selecionado, revista publicada, base de dados, autor (es) e resumo breve. Dando sequência, a quarta etapa se envolveu através da leitura dos estudos incluídos e, para finalizar a seleção, a quinta etapa constituiu na interpretação e discussão desses estudos, chegando a escrita da revisão integrativa. 
Figura 1: Artigos selecionados.

\begin{tabular}{|c|c|c|c|c|c|}
\hline Ano & Estudo selecionado & $\begin{array}{c}\text { Revista } \\
\text { publicada }\end{array}$ & Base de dados & Autores & Resumo \\
\hline 2015 & $\begin{array}{c}\text { O desafio do consolo: } \\
\text { vivências do enfermeiro } \\
\text { com o cuidado espiritual } \\
\text { e existencial do } \\
\text { moribundo - um estudo } \\
\text { fenomenológico- } \\
\text { hermenêutico. }\end{array}$ & BMC Nurs & PUBMED & $\begin{array}{c}\text { Kirsten Anne } \\
\text { Tornøe, Lars } \\
\text { Johan Danbolt, } \\
\text { Kari Kvigne, } \\
\text { Venke Sørlie. }\end{array}$ & $\begin{array}{c}\text { Entrevistas individuais } \\
\text { realizadas com } \\
\text { enfermeiras que se } \\
\text { esforçaram para ajudar } \\
\text { os pacientes a aceitar a } \\
\text { morte, resolver questões } \\
\text { práticas e alcançar a } \\
\text { reconciliação com seu } \\
\text { passado. }\end{array}$ \\
\hline 2017 & $\begin{array}{c}\text { Empatia, solidão, } \\
\text { esgotamento e satisfação } \\
\text { com a vida em } \\
\text { enfermeiras chilenas de } \\
\text { cuidados paliativos e } \\
\text { serviços domiciliares. }\end{array}$ & Enferm Clin & PUBMED & $\begin{array}{l}\text { Magdalena } \\
\text { Marilaf Caro, } \\
\text { Montserrat San- } \\
\text { Martín, Roberto } \\
\text { Delgado-Bolton, } \\
\text { Luis vivanco. }\end{array}$ & $\begin{array}{c}\text { As evidências } \\
\text { encontradas sugerem que } \\
\text { as habilidades empáticas } \\
\text { podem ser aprimoradas } \\
\text { com a experiência } \\
\text { profissional }\end{array}$ \\
\hline 2018 & $\begin{array}{c}\text { Atendendo às } \\
\text { necessidades de } \\
\text { pacientes em estado } \\
\text { terminal na Bósnia- } \\
\text { Herzegovina: } \\
\text { percepções e } \\
\text { expectativas dos } \\
\text { pacientes. }\end{array}$ & $\begin{array}{c}\text { BMC Palliat } \\
\text { Care }\end{array}$ & PUBMED & $\begin{array}{c}\text { S Aebischer } \\
\text { Perone , R } \\
\text { Nikolic , R } \\
\text { Lazic , E Dropic } \\
\text {, T Vogel , B } \\
\text { Lab , S Lachat, } \\
\text { P Hudelson, C } \\
\text { Matis , S Pautex } \\
\text {, F Chappuis. }\end{array}$ & $\begin{array}{c}\text { Resultados sugerem uma } \\
\text { espaço entre as cuidados } \\
\text { de fim de vida dos } \\
\text { pacientes e os serviços } \\
\text { existentes. O estudo } \\
\text { mostra a importância em } \\
\text { fornecer apoio físico mas } \\
\text { também e psicossocial }\end{array}$ \\
\hline
\end{tabular}

Fonte: Autores, (2021).

\section{Resultados}

Buscando analisar os seguintes critérios: área de conhecimento, variáveis, metodologia utilizada e relevância do estudo; foram encontrados 54 artigos, após aplicação dos critérios de inclusão restaram 15 estudos, porém, somente 3 foram definidos para a revisão por preencherem os critérios de seleção. Os resultados dessa busca foram organizados na figura 2 abaixo, incluindo, de maneira quantitativa, os artigos selecionados em cada etapa. 
Figura 2 - Estratificação dos resultados encontrados.

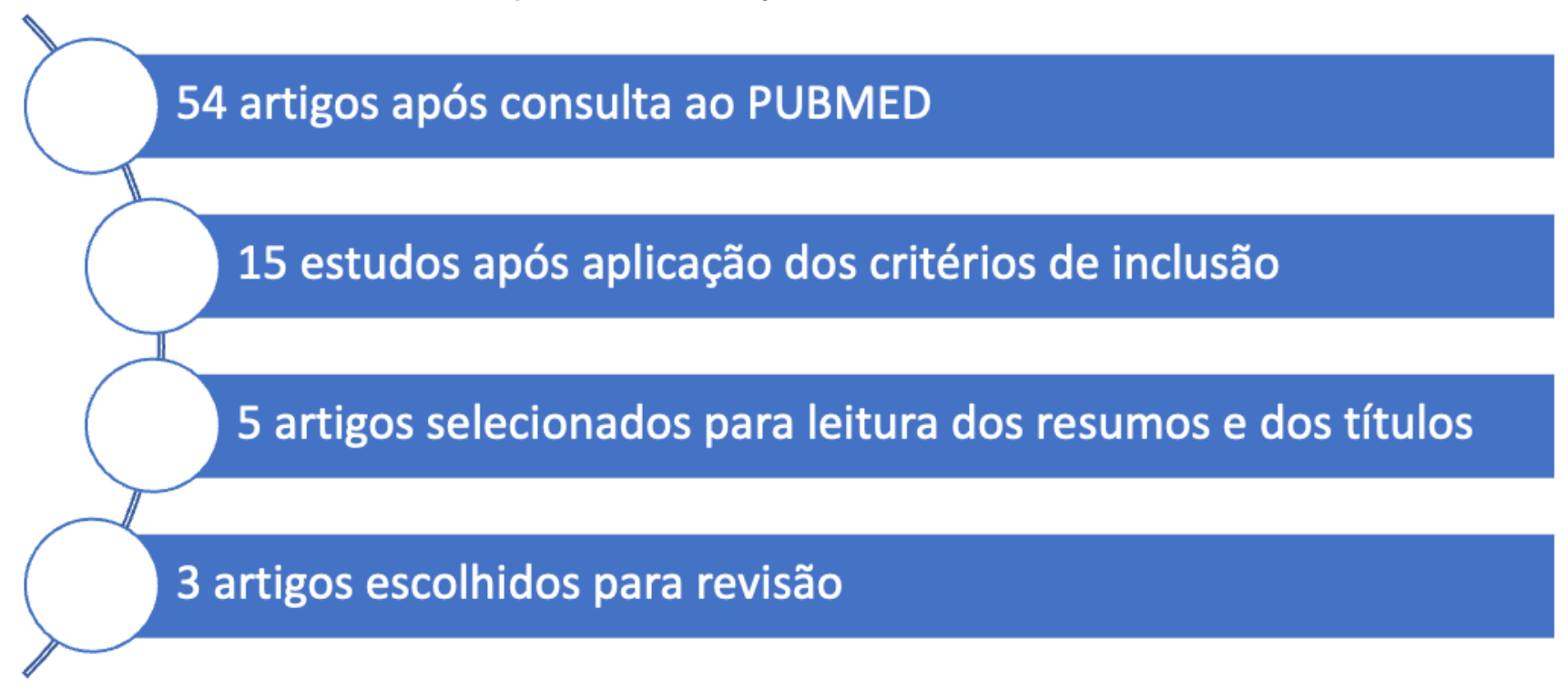

Fonte: Autores, (2021).

\section{Discussão}

Após o levantamento da literatura, foi realizada uma análise dos artigos escolhidos, e a temática foi separada em 3 categorias: causas da dor social, qualidade de vida dos pacientes oncológicos elegíveis aos cuidados paliativos e as ações dos profissionais de saúde frente à dor social.

\section{Causas da dor social}

As principais causas da dor social são solidão e mudança no convívio social, outros motivos/causas como angústia diante da finitude da existência humana e a visão dos pacientes de querer poupar os entes queridos do sofrimento em relação a doença podem levar também a esse tipo de sofrimento (Crofford, 2015).

A angústia diante da finitude traz ao paciente problemas para encontrar sentido em sua vida e em aceitar a doença, muitas vezes acreditando ser um pecador que recebe um castigo. $\mathrm{O}$ isolamento como forma de poupar a família do sofrimento se faz bastante presente à medida que a doença caminha para fora de possibilidade curativas, por muitas vezes os pacientes manifestam ansiedade e insegurança pois, eles têm preocupações em relação ao futuro de seus entes queridos. Esse distanciamento contribui para o sentimento de solidão e desamparo tanto para o paciente quanto para a família (Campos, Zhang, Sinclair, Tsao, Barnes, Danjoux, Sahgal, Goh, Culleton, Mitera \& Chow, 2009).

\section{Qualidade de vida dos pacientes oncológicos elegíveis aos cuidados paliativos}

Cerca de 63\% dos pacientes declaram estar com depressão, principalmente por causas de isolamento e de solidão. Quanto mais dependentes em suas atividades de vida diária mais deprimidos se sentem. Enfrentar o agravamento de sua condição clínica e a chegada da morte iminente junto aos sintomas dolorosos foram agravantes da depressão. Em suas oratórias eles expressam não estarem bem e desejam que a morte ocorra em breve e relatam chorar o tempo todo (Perone, Nokolic, Lazic, Dropic, Vogel, Lab, Lachat, Hudelson, Matis, Pautex, \& Chappuis, 2018).

A depressão é um problema de saúde pública que afeta milhares de pessoas. Há uma prevalência maior em mulheres do que em homens independentemente da faixa etária, isso pode ser atribuído a diversos fatores como variações hormonais, viver mais que os homens, vivenciar eventos mais traumáticos, como luto e o ninho vazio. Vale ressaltar que os laços 
familiares podem ajudar com as consequências indesejáveis associadas à depressão (Didoné, Jesus, Orlandi, Pavarini, Orlandi, Guarisco, Gratão, Say, Cominetti, Gomes \& Zazzetta, 2020).

A necessidade de apoio psicológico e tratamento contra a ansiedade foi enfatizado em diversas situações. A capacidade de aproveitar a vida poderia ser resgatada com o aumento da interação social, mais visitas ao domicilio e até com interações tecnológicas (Hennemann-Krause, 2012). O simples fato de sair da cama ou de casa foi considerado como fonte de alívio. Em relação a qualidade de vida dos pacientes elegíveis aos cuidados paliativos o estado de saúde global e função desempenho de papel tiveram pior avaliação. Na escala de sintomas, a dor, fadiga, insônia e perda do apetite tiveram destaque (Figueiredo, Souza, Coelho \& Souza, 2018).

É importante ressaltar que a idade avançada deve ser analisada especialmente nesses indivíduos, o declínio na capacidade funcional, motora, modificações no metabolismo e diversas doenças crônicas em associação são fatores que devem ser observados com cuidado, pois as modificações associadas às reações impostas pelas diversas formas de tratamento do câncer, provocam uma sensação de fadiga crescente, perda de peso e redução da força muscular, que comprometem a capacidade funcional do indivíduo e qualidade de vida (Frossard, Rodrigues, Oliveira \& Fernandes, 2018). INCA, 2020

O Brasil é o segundo país da América Latina em que pessoas com câncer relatam sentir mais o sintoma da dor. E com o avanço do câncer, os pacientes relatam uma diversidade de sinais e sintomas que afetam de forma significativa a sua qualidade de vida. Compreender que a dor gera um intenso desconforto físico e psicológico impõe que profissionais de saúde ofereçam uma promoção imediata do alívio do sintoma, que para muitos pode se tornar insuportável e afeta negativamente qualidade de vida do paciente. (Freire, Costa, Lima \& Sawada, 2018).

\section{Ações dos profissionais de saúde frente à dor social}

É imprescindível que os profissionais da saúde saibam como agir diante desses pacientes, que querem ser tratados como pessoas e não como doenças, serem observados como um todo incluindo aspectos físicos, emocionais, sociais e espirituais (Bosi, 2012). As enfermeiras tentam intervir com o intuito de trazer uma vida/morte pacífica e harmoniosa a esses pacientes, ajudar a aceitar a morte e encontrar reconciliação são atitudes recorrentes. Elas dizem que não se sentem totalmente preparadas para atuar nas questões psicológicas/espirituais desses pacientes, e o sofrimento social continua a ser um grande desafio para os profissionais de saúde. O sofrimento físico é mais bem atendido que o sofrimento social, percebe-se então que se faz necessário o conhecimento desse sofrimento, avaliação e intervenção corretas (Freire, Costa, Lima \& Sawada, 2018).

Criar vínculo, estabelecer confiança, analisar os níveis de funcionalidade e o estado emocional desses pacientes são medidas cruciais para a prevenção e alívio do sofrimento social (Monteiro, Almeida \& Kruse, 2013).

\section{Considerações Finais}

As causas da dor social envolvem as mudanças nas atividades de vida diária, troca de papéis sociais, isolamento e solidão. Uma vez que estes pacientes se encontram em fase final de vida, torna-se um desafio para manter sua qualidade de vida pelo sentimento recorrente de abandono. Sendo assim, os cuidados paliativos aliados a atenção domiciliar representam uma ferramenta chave para a abordagem desses pacientes e o alívio e prevenção da dor social.

Assim, ações educativas e preventivas relacionadas ao cuidado adequado a esses pacientes são de extrema importância nessa fase final de vida. Espera-se que os cuidados paliativos sejam abordados com mais atenção nas universidades para que se consiga resultados mais significativos no tratamento desses pacientes, sejam eles cuidados domiciliares ou em qualquer instituição de saúde. 
Como trabalhos futuros, almeja-se a realização de mais estudos que abordem esse tema, com o objetivo de preparar de forma mais eficiente e qualificada todos os profissionais de saúde que prestam esse tipo de atendimento, levando uma melhor qualidade de vida a todos os pacientes oncológicos.

\section{Limitações}

Este estudo é limitado por considerar apenas artigos em Português, Inglês e Espanhol e por não abranger outras bases de dados.

\section{Referências}

Aktas, D. \& Terzioglu, F. (2012). Effect of Home Care Service on the Quality of Life in Patients with Gynecological Cancer. Asian Pac J Cancer Prev, 16 (9), 4089-4094

Bosi, M. L. M. (2012). Pesquisa qualitativa em saúde coletiva: panorama e desafios. Ciência e saúde coletiva. 17(3), p. 575-586.

Campos, S., Zhang, L., Sinclair, E., Tsao, M., Barnes, E.A., Danjoux, C., Sahgal, A., Goh, P., Culleton, S., Mitera, G. \& Chow, E.. (2009). The palliative performance scale: examining its inter-rater reliability in an outpatient palliative radiation oncology clinic. Support Care Cancer. 17(6):685-90

Crofford, L. J. (2015). Chronic Pain: Where the Body Meets The Brain. Trans Amer Clin and Climat Assoc. 126:167-81

Didoné, L. S., Jesus, I. T. M., Orlandi, A. A. S., Pavarini, S. C. I., Orlandi, F. S., Guarisco, L. P. C., Gratão, A. C. M., Say, K. G., Cominetti, M. R., Gomes, G. A. O. \& Zazzetta, M. S. (2020). Factors associated with depressive symptoms in older adults in context of social vulnerability. Revista Brasileira de Enfermagem. 73(1).

Figueiredo, J. F., Souza, V. M. S., Coelho, H. V. \& Souza, R. S. (2018). Qualidade de Vida de Pacientes Oncológicos em Cuidados Paliativos.

Frossard, A., Rodrigues, L., Oliveira, B. \& Fernandes, C. (2018). Serviço Social e Oncologia: os Cuidados Paliativos em foco. E-revista.

Freire, M. E. M., Costa, G. F. S., Lima, G. A. R. \& Sawada, M. O. (2018). Qualidade de vida relacionada à saúde de pacientes com câncer em cuidados paliativos. Texto e contexto - enfermagem. 27(2).

Hennemann-Krause, L. (2012). Dor no Fim da Vida: Avaliar para Tratar. Revista Hospital Universitário UERJ.11:26, 31-32.

Mendes, K. D. S., Silveira, R. C. de C. P., \& Galvão, C. M. (2008). Revisão integrativa: método de pesquisa para a incorporação de evidências na saúde e na enfermagem. Texto \& Contexto - Enfermagem, 17(4), 758-764. DOI: 10.1590/s0104-07072008000400018

Ministério da Saúde. DATASUS. (2019). Mortalidade Geral no Brasil por Neoplasias.

Ministério da Saúde. INCA. O que é câncer? (2019).

Ministério da Saúde. INCA. Estimativa de Câncer no Brasil, 2020.

Monteiro, D. R., Almeida, M. A. \& Kruse, M. H. L. (2013). Tradução e adaptação transcultural do instrumento Edmonton Symptom Assessment System para uso em cuidados paliativos. Revista Gaúcha Enfermagem. 34(2).

Perone, S. A., Nokolic, R., Lazic, R., Dropic, E., Vogel, T., Lab, B., Lachat, S., Hudelson, P., Matis, C., Pautex, S. \& Chappuis, F. (2018). Addressing the needs of terminally-ill patients in Bosnia-Herzegovina: patients' perceptions and expectations. BMC Palliat Care. 17(1):123

Roque, T. da S., Silva, B. T. da, Santos, C. S., Sousa, J. I. dos S. \& Perim, L. F. (2020). Palliative care in elderly people: a literature review. Research, Society and Development. S. l.(9). DOI: 10.33448/rsd-v9i4.3010.

Sarmento, V. P., Gysels, M., Higginson, I. J. \& Gomes, B. (2017). Home palliative care works: but how? A meta-ethnography of the experiences of patients and family caregivers. BMJ Supportive \& Palliative Care. 390-403.

Souza, K. R. \& Kerbauy, M. T. M. (2017). Abordagem quanti-qualitativa: superação da dicotomia quantitativa-qualitativa na pesquisa em educação. Educação e Filosofia. 31(61), p. 21-44.

Vasconcelos, G. B. \& Pereira, P. M. (2018) Cuidados paliativos em atenção domiciliar: uma revisão bibliográfica. Revista de Administração em Saúde. 18(70).

Waterkemper, R. \& Reibnitz, K. S. (2010). Cuidados Paliativos: A avaliação da dor na percepção de enfermeiras. Revista Gaúcha Enfermagem. 31(1):84-91.

World Health Organization. (2016). Definition of Palliative Care, 